\title{
A Case of Esophageal Candidiasis in an Adolescent Who Had Received Repeated Courses of Brucellosis Therapy
}

\author{
Mohamed Attiya ${ }^{1}$ and Abeer Fathy ${ }^{2 *}$ \\ ${ }^{1}$ Department of internal medicine, Mansoura University, Egypt \\ ${ }^{2}$ Department of Pediatrics, Mansoura University, Egypt, Department of Pediatrics, Taibah University, KSA, Saudi Arabia
}

*Corresponding author: Abeer Fathy, Department of Pediatrics, Mansoura University, Egypt and Department of Pediatrics, Taibah University, KSA, Saudi Arabia, Tel: +966530530686, E-mail: abeerfathy2000@yahoo.com

Citation: Mohamed Attiya, Abeer Fathy (2018) A Case of Esophageal Candidiasis in an Adolescent Who Had Received Repeated Courses of Brucellosis Therapy. Saj Case Rep 5: 406

Article history: Received: 11 August 2018, Accepted: 24 September 2018, Published: 26 September 2018

\begin{abstract}
The standard drug therapy for brucellosis is doxycycline-rifampin (DR) regimen for six weeks. Such treatment regimen is generally well tolerated and does not cause any severe complications that necessitate discontinuation of medication. The authors present a case of esophageal candidiasis in a healthy adult male who was treated with two consecutive courses of doxycycline-rifampin therapy. This patient presented with painful swallowing for 1 month. Esophageal endoscopy showed a whitish creamy pseudomembrane and erosions on the esophageal mucosa. Pathologic findings showed numerous candidal hyphae. The patient has no evidence of immunodeficiency, clinically and historically. The esophageal lesion completely improved with the antifungal therapy for 2 weeks; the symptoms disappeared, and the patient returned to normal health.
\end{abstract}

Keywords: Esophagus; Candidiasis; Doxycycline; Rifampicin

\section{Introduction}

Brucellosis is an important zoonotic disease that has a wide range of manifestations in man from an acute febrile illness to mild nonspecific complaints, and its duration varies from several days to several years [1]. Treatment of brucellosis is best achieved with combination drug therapy that leads to better recovery of symptoms, shortening of symptoms duration, decrease in the relapse episodes and lower rate of drug resistance [2]. The WHO has recommended a doxycycline-rifampin (DR) regimen for six weeks as the standard drug therapy for brucellosis in man [3]. Treatment with doxycycline (100 mg PO twice daily) and rifampicin $(15 \mathrm{mg} / \mathrm{Kg}$ body weight as a single daily dose) is generally well tolerated and does not cause any severe complications that necessitate discontinuation of medication. Common side effects reported with doxycycline treatment include loss of appetite, nausea and vomiting, diarrhea, rash, sensitivity to the sun and hives. Serious side effects that may occur with doxycycline therapy include antibiotic associated diarrhea, esophageal ulceration, esophagitis, anemia and pancreatitis [4]. Side effects associated with rifampicin include gastrointestinal upsets, diarrhea and pseudomembranous colitis, transient abnormalities in liver function tests, thrombocytopenia, leukopenia, and hemolytic anemia. Nervous symptoms as headache, ataxia, dizziness, generalized numbness and visual disturbances have been also observed [5].

\section{Case report}

A 17-year-old male patient presented to gastroenterology clinic complaining of painful swallowing that started over the last month and became aggravated 2 weeks ago. The pain occurred intermittently with swallowing of both liquid and solids. It was associated with loss of appetite, no fever or significant loss of body weight. Three months ago, the patient had prolonged fever which was diagnosed as Brucellosis based on suggestive history and positive serology; he was given a course of Doxycycline and Rifampicin. After initial improvement, relapse of symptom occurred due to poor compliance on medication. The patient was given another 6 weeks course of Doxycycline and Rifampicin to which the patient was strictly compliant. That patient did not receive any other medication and he has no other chronic illnesses. To investigate for this patient dysphagia, upper gastrointestinal endoscopic examination was done. The whole esophageal mucosa was covered with creamy white pseudo-membranes and had multiple erosions, the gastric and duodenal mucosa were normal. Biopsies were taken from the esophageal lesions for pathologic studies; $\mathrm{KOH}$ staining showed hyphae that suggested a fungal infection. Hematoxylin and eosin staining showed numerous hyphae and 
yeasts on the surface of the hyperkeratotic squamous epithelium of the esophagus. The diagnosis of esophageal candidiasis was confirmed (Figure 1).
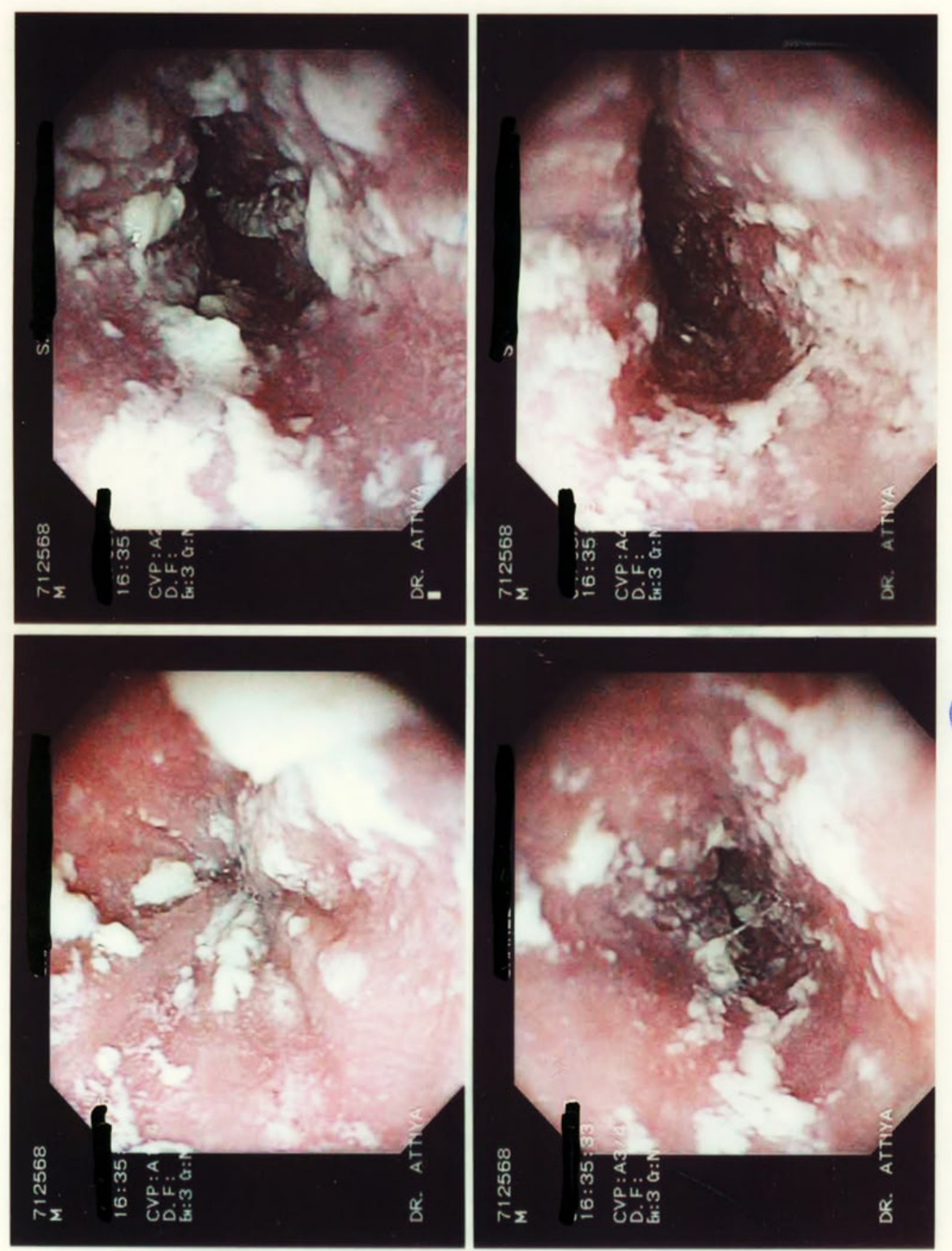

Figure 1: Endoscopic findings of the reported case

The patient's medical history and clinical examination were suspicious for any immunocompromised state. However, we did a full laboratory work up to rule out any hidden cause. Blood studies were done; they showed WBC 5,600/mm3 (polymorphonuclear neutrophils $52 \%$, lymphocyte $38 \%$, monocyte $7 \%$ ), hemoglobin $12.4 \mathrm{~g} / \mathrm{dL}$, platelet $255,000 / \mathrm{mm} 3$. C-reactive protein was negative. Electrolytes, total protein, albumin, blood glucose level, liver enzymes and renal profiles were all normal. In addition, CD3 and CD4 were within the normal range and human immunodeficiency virus serology was negative.

The patient was treated with oral fluconazole (200 mg orally on the first day followed by $100 \mathrm{mg}$ orally once daily for 3 weeks). The patient showed gradual improvement of his symptoms and clinical condition as he took the medicine. A follow-up esophagoscopic examination was performed 2 weeks after the cessation of the anti-fungal therapy. The whitish creamy pseudo-membranes and multiple erosions were no longer seen in theesophagus. The esophageal mucosa was normal.

\section{Discussion}

Esophageal candidiasis (EC) is one of the most common opportunistic infections in patients with impaired immunity, such as those with human immunodeficiency virus (HIV) infection, prolonged neutropenia and immunodeficiency diseases affecting neutrophil functions [6]. When EC occurred in healthy individuals without HIV infection, predisposing medical conditions have often been identified [7]. Cases of EC have been reported with drug-induced suppression of gastric acid production as 
with prolonged therapy with $\mathrm{H}_{2}$-receptor antagonists or Proton-pump inhibitors [8,9]. Some studies reported that corticosteroid and cytotoxic drugs are also possible risk factors of EC [10]. Esophageal disease, such as noninfectious esophagitis or achalasia may favor the development of EC [11]. Other risk factors for esophageal candidiasis include diabetes mellitus, hypothyroidism, hypoparathyroidism, malnutrition, chronic infectious disease, alcoholism, and advanced age [11].

The use of Broad-spectrum antibiotics is a well-known risk factor for EC; it eliminates certain bacteria that inhibit fungal growth, thereby enhancing candida overgrowth. Most of the reported cases were for patients receiving streptomycin, tetracycline or penicillin for two to four weeks [12]. Drug induced esophagitis and ulceration are well- known complications of Doxycycline [13]. Doxycycline causes direct damage to esophageal mucosa. Factors such as chemical contents, duration of contact to mucosa, volume of the capsule, gelatin cover and individual factors such as esophageal motility disorder and swallowing without water may affect the degree of Doxycycline induced esophagitis [14]. In a retrospective analysis of 48 cases with proved diagnosis of doxycycline- or tetracycline-induced esophageal injury by endoscopy, Gencosmanoglu and his colleagues reported that the pattern of esophageal damage is related to the type of tetracycline used by patients [15]. They found that Doxycycline was more frequently associated with mid-esophageal ulceration while Tetracycline was associated with distal esophagitis with or without candidiasis.

In our case, a previously healthy immune competent patient had diffuse candidiasis and ulceration affecting the whole esophagus following 6 weeks course of doxycycline therapy. Up to our knowledge this is the first reporting of such pattern of affection with doxycycline therapy.

\section{References}

1. Harrison TR, Madkur MM, Kasper DL (2001) in: Harrison’s Principles of Internal Medicine. (15th edn). McGraw-Hill, New York 1: 986-91.

2. Acocella G, Bertrand A, Beytout J, Durrande JB, Garcia Rodriguez JA, et al. (1989) Comparison of three different regimes in the treatment of acute brucellosis: a multicenter multinational study. J Antimicrob Chemother 23: 433-9

3. Mandell GL, Benett JE, Dolin R (2005) in: Principles and Practice of Infectious Disease. (6 ${ }^{\text {th }}$ edn) Churchill Livingstone, London 13: $2669-674$.

4. Watts TL (2003) Significant complications of doxycycline. Br Dent J 195: 65.

5. Akbar DH, Al-ShehriHZ, Al-HuzaliAM, Falatah HI (2003) A case of rifampicin induced pseudomembraneous colitis. CaseRepClinPractRev 4: 338-41

6. Misra S, Ament ME. Esophagitis. In: Feigin RD, Cherry JD, eds. (1997) Textbook of pediatric infectious diseases. (4 ${ }^{\text {th }}$ edn). Philadelphia: Saunders 562.

7. Kliemann DA, Pasqualotto AC, Falavigna M, Giaretta T, Severo LC (2008) Candida esophagitis: species distribution and risk factors for infection. Rev Inst Med Trop Sao Paulo 50: 261-3.

8. Kochhar R, Talwar P, Singh S, Mehta SK (1988) Invasive candidiasis following cimetidine therapy. Am J Gastroenterol 83: 102-3.

9. Karmeli Y, Stalnikowitz R, Eliakim R, Rahav G (1995) Conventional dose of omeprazole alters gastric flora. Dig Dis Sci 40: 2070-3.

10. Simon MR, Houser WL, Smith KA, Long PM (1997) Esophageal candidiasis as a complication of inhaled corticosteroids. Ann Allergy Asthma Immunol 79: 333-8.

11. Phaosawasdi K, Rice P, Lee B (1986) Primary and secondary Candida esophagitis. IMJ Ill Med J 169: 361-5

12. Thapa BR, Kumar L (1989) Candida esophagitis after antibiotic use. Indian J Pediatr. 56: 296-9.

13. Arısı DS, Göze F, Akbayır N (1999) Doxycyline-induced esophagitis. Turk J Gastroenterol 10: 1-3.

14. Agha FB, Wilson JA, NLostrand TT (1986) Medication induced esophagitis. GastrointestRadiol 11: 7-11.

15. Gencosmanoglu R1, Kurtkaya-Yapicier O, Tiftikci A, Avsar E, Tozun N, et al. (2004) Mid-esophageal ulceration and candidiasis-associated distal esophagitis as two distinct clinical patterns of tetracycline or doxycycline-induced esophageal injury. J Clin Gastroenterol 38: 484-9. 